\title{
The Surgical Strategy to Correct the Rotational Imbalance of the Glenohumeral Joint after Brachial Plexus Birth Injury
}

\author{
J. Bahm ${ }^{1}$ \\ ${ }^{1}$ Euregio Reconstructive Microsurgery Unit, Franziskushospital, \\ Aachen (D), Germany \\ J Brachial Plex Peripher Nerve Inj 2016;11:e10-e17.
}

\begin{abstract}
Keywords

- brachial plexus birth injury

- medial rotation contracture

- glenohumeral dysplasia

- suprascapular nerve

- nerve transfer

- muscle transfer

- humeral osteotomy

In upper brachial plexus birth injury, rotational balance of the glenohumeral joint is frequently affected and contracture in medial rotation of the arm develops, due to a severe palsy or insufficient recovery of the lateral rotators. Some of these children present with a severe glenohumeral joint contracture in the first months, although regular physiotherapy has been provided, a condition associated with a posteriorly subdislocated or dislocated humeral head. These conditions should be screened early by a pediatrician or specialized physiotherapist. Both aspects of muscular weakness affecting the lateral rotators and the initial or progressive glenohumeral deformity and/or subdislocation must be identified and treated accordingly, focusing on the reestablishment of joint congruence and strengthening of the lateral rotators to improve rotational balance, thus working against joint dysplasia and loss of motor function of the shoulder in a growing child. Our treatment strategy adapted over the last 20 years to results from retrospective studies, including biomechanical aspects on muscular imbalance and tendon transfers. With this review, we confront our actual concept to recent literature.
\end{abstract}

\section{Introduction}

The rotational balance of the glenohumeral joint $(\mathrm{GHJ})$ is assumed by a dynamic equilibrium between two antagonistic muscle groups: the lateral rotators (supraspinatus and overall infraspinatus muscle, innervated by the suprascapular nerve exiting the upper trunk, and the teres minor muscle, innervated by the axillary nerve originating from the posterior cord) and the medial rotators (mainly the subscapularis muscle, but also the conjoined tendon of the latissimus dorsi and teres major muscle, all innervated by selective motor branches arising from the posterior cord).

In an upper (affecting the roots C5 and C6) and extended upper (affecting C5, C6, and C7) brachial plexus birth injury (BPBI), the motor nerves of both lateral and medial rotators are traumatized. However, clinical experience shows a dominant lack of recovery of the infraspinatus muscle, while the medial

received

June 18, 2014

accepted

January 22, 2016
DOI http://dx.doi.org/ 10.1055/s-0036-1579763. ISSN $1749-7221$. rotation and the subscapular muscle recover faster and better. Thus, the arm is positioned or maintained in medial rotation. This situation may become functionally compromising when progressing to a medial rotation contracture (MRC), reducing the passive range of motion (ROM) of the $\mathrm{GHJ}$ in lateral rotation (LR).

This muscular imbalance may be treated first by regular passive stretching exercises, performed by a specialized physiotherapist who also teaches the parents, if the passive ROM of the GHJ is maintained within normal limits while awaiting the recovery of active LR (aLR). Nevertheless, a typical MRC develops frequently with progressive decline of the passive range of LR in the GHJ with the arm in adduction. In a severe MRC, ultrasound or magnetic resonance imaging (MRI) of the GHJ shows variable signs of progressive glenohumeral dysplasia (GHD): the humeral head is located in posterior subdislocation, the shape of the head might be irregular, and retroversion is enhanced. The 
glenoid itself is either concave, or flattened, or convex, or develops a posterior pseudoglenoid with a second cavity. ${ }^{1,2}$

The pathologic neuro-orthopedic sequence behind MRC starts with a complex nerve lesion at the upper trunk (-Fig. 1) leading to various degrees of weakness in the antagonistic muscle groups for shoulder and elbow motion. This palsy together with bone and joint deformities in the growing $\mathrm{GHJ}$ is actually recognized as a severe and frequent sequel, associated with upper and extended upper BPBI lesions, regardless of having undergone nerve reconstruction or not. It is rare in total BPBI lesions, related to the poor recovery of the subscapular muscle in these cases.

Recent papers ${ }^{3-9}$ update the common knowledge on this problem and present postoperative results with variable follow-up periods.

In this review, we explain the conceptual development of our own treatment strategy build up since 1994 and confront it to the actual literature. We also stress the need to specify in any MRC treatment strategy which particular shoulder movement is considered for improvement, either in pre- and postoperative LR assessment or when performing muscle transfers aimed to improve shoulder LR.

Muscular imbalance also plays a role in other sequels from $\mathrm{BPBI}$, such as the anterior radial head subdislocation associated with an impaired prosupination resulting in a rotational disbalance around the forearm longitudinal axis. ${ }^{10}$

\section{Pathophysiology}

Most of the knowledge concerning MRC development is based on regular clinical experience and empiric intraoperative

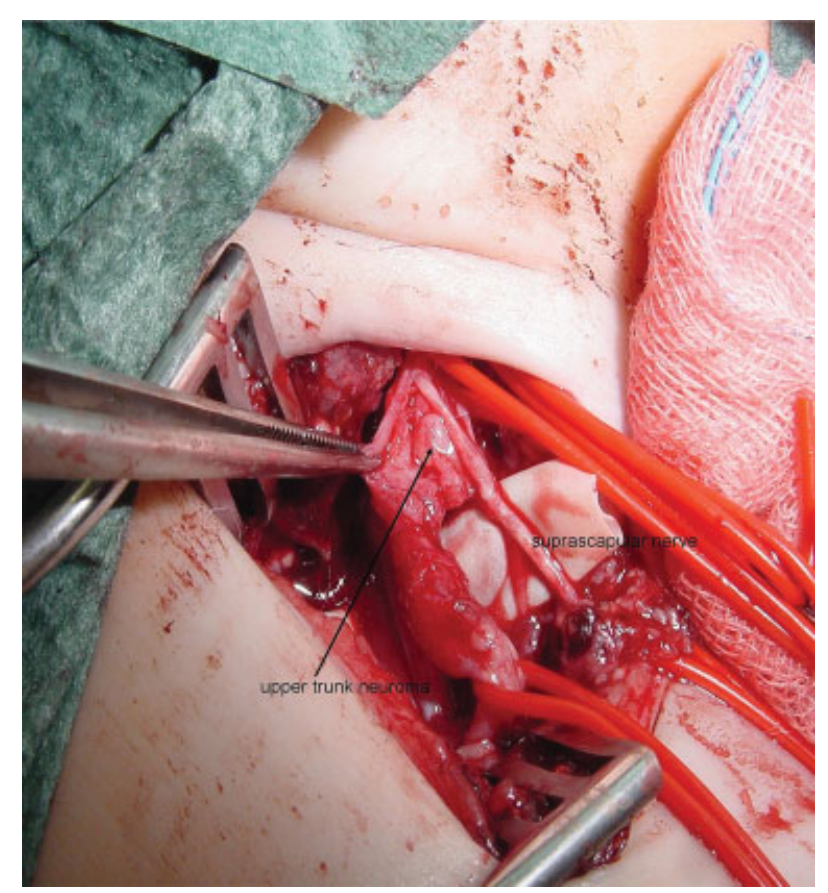

Fig. 1 Intraoperative view of an upper BPBI lesion through a supraclavicular approach: the suprascapular nerve exiting the upper trunk is shown. In an upper trunk neuroma, this emergence is frequently scarred and might explain the bad recovery of this nerve under these circumstances in an Erb palsy. observation, as neither animal experimental models, ${ }^{11}$ nor histopathology of subscapular muscle, ${ }^{12}$ nor MRI studies investigating muscle atrophy and GH deformity ${ }^{13}$ are conclusive. Muscular imbalance between antagonistic lateral and medial rotators seems to be a universally accepted background to this entity. Thus, the treatment in the young is aimed to restore the function of the suprascapular nerve, either by physiotherapy accompanying the nerve regeneration process, or by nerve repair (using an isolated graft or a nerve transfer), or by temporary weakening of the subscapular muscle using botulinum toxin.

-Fig. 1 reminds how the emergence of the suprascapular nerve, arising out of the distal upper trunk, is critical in upper root neuroma, where only little damage or scarring within this emergence area could easily compromise the proper reinnervation of the proximal suprascapular nerve. Moreover, little is known about the existence of second-level injuries, for example, at the scapular notch (which could explain some bad results even after minor neuroma in the trunk or after proximal nerve transfers). It is mandatory to consider this selective primary nerve injury, either isolated or as part of an upper or total BPBI, in the context of progressive MRC and thus concentrate on a proper suprascapular nerve recovery. This direct nerve repair might be complemented later on by a muscle slide or lengthening tenotomy for the too strong subscapular muscle $\mathrm{e}^{14,15}$ or a tailored muscle-tendon transfer onto the rotator cuff to increase the power of aLR. ${ }^{16}$

The GHJ incongruence regularly appears as a delayed progressive phenomenon, thus seeming secondary to the muscular imbalance, but may be present as early as within the first months of life. It has been clearly documented before 1 year of age, 1,17,18 but seems to be rare. In a prospective study, $7 \%$ of all BPBI suffered from a posterior shoulder dislocation before 1 year. ${ }^{17}$ Any peripartal direct trauma to the humeral head could end in a potentially unrecognized peripartal (neonatal) glenohumeral (sub)dislocation, ${ }^{19}$ which needs an immediate orthopedic strategy, including a closed humeral head reposition and a plaster immobilization in LR with the arm in adduction.

The knowledge about the sequence of deformation of the $\mathrm{GH}$ contact area is scarce. Biomechanic considerations paralleling studies about the hip joint done by Pauwels ${ }^{20,21}$ were detailed in our thesis, ${ }^{2}$ where we analyzed the pathologic force vectors which probably contribute to the deformities observed clinically, and especially the backward movement of the humeral head parallel to MRC (-Fig. 2). For the last 40 years, the development and growth of a joint with its constitution of bone, cartilage, and connective tissue (capsule, ligaments, and tendons) under evolving forces, torques, and pressure constraints have been well described by Pauwels using the concept of "functional histogenesis." ${ }^{22}$ We lack clear data stating how long the $\mathrm{GHJ}$ of a child is deformable in a way to reverse a pathologic dysplasia initiated by MRC, but clinical evidence agrees about an approximate 2 years' limit.

\section{Surgical Strategy and Patient Evaluation}

Based on these considerations, we conducted a retrospective study on GH deformation in 50 consecutive children 


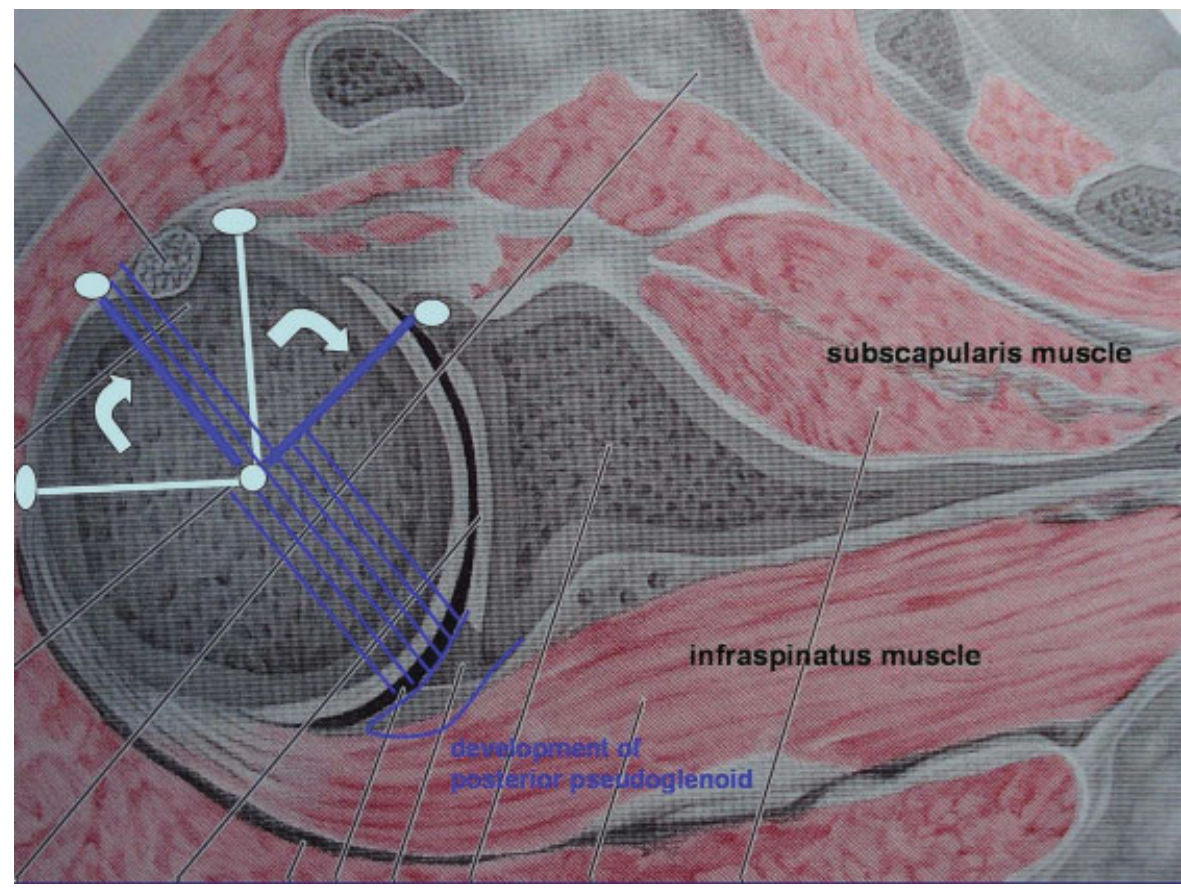

Fig. 2 Pathological forces onto the GHJ in MRC. The arrows show the MR of the humeral head, leading to a posteriorly oriented force line producing the deformity of the posterior glenoid.

consulting for BPBI-related MRC ${ }^{1}$ and another on the functional outcome after elective or combined nerve transfer to reinnervate the suprascapular nerve, ${ }^{23}$ eventually as an isolated primary or a secondary surgery using a limited dorsal approach. ${ }^{24}$ With these results, we progressively established our treatment strategy as summarized in -Table 1. The rationale is an age-dependent set of procedures, aiming first to restore joint congruence and improve passive LR (pLR) of the GHJ, investigating in parallel the recovery of the suprascapular nerve (thereby considering the indication for a selective nerve transfer). Tendon transfers aimed to increase active motion are indicated in an early but second line. We refuse combinations between shoulder release and tendon transfer surgery.

The type of muscle-tendon unit(s) transfer is chosen according to the individual motion needs of the child in different planes. The pre- and postoperative evaluation of aLR and pLR (-Table 2) has to separate any movement in a horizontal or vertical plane while the arm is, respectively, adducted or abducted ( - Figs. 3 and $\mathbf{4}$ ), respecting Blaauw and Muhlig's comment about the range of aLR and the neutral position. ${ }^{25}$

LR of the arm is performed through the GHJ. Biomechanics are different when the arm is in neutral position, along the chest, in adduction ( - Fig. 3; LR[ADD]), where the rotational axis is vertical, compared with an arm in 90-degree abduction, in the sagittal plane, where the rotational axis is horizontal (-Fig. 4; LR[ABD]). aLR(ADD) requires a muscle action line perpendicular to the (vertical) rotation axis by a shoulder muscle which is oriented horizontally (the spinati with the scapula at rest; -Fig. 5), whereas aLR(ABD) is executed by a vertical muscle pull (the spinati with a tilted scapula; - Fig. 6). Any muscle transfer augmenting rotational forces must thus be oriented horizontally to improve aLR (ADD) and vertically to improve aLR(ABD) ( - Fig. 7). Of course, between these two extreme positions, a lot of intermediate sets with specific muscle force vectors exist.

Table 1 Treatment strategy in medial rotation contracture (MRC)

\begin{tabular}{|c|c|}
\hline & Treatment strategy \\
\hline Early (first months of live) MRC with subdislocated humeral head & Closed reposition and plaster in pLR(ADD) \\
\hline Early progressive MRC with congruent humeral head & $\begin{array}{l}\text { Physiotherapy, eventually botulinum toxin into the } \\
\text { subscapularis muscle }\end{array}$ \\
\hline MRC before 2 y not responding to physiotherapy & Subscapular slide or anterior release surgery \\
\hline Lack of aLR(ABD) between 6 and $18 \mathrm{mo}$ & SSC nerve neurotization \\
\hline Lack of aLR(ABD) or aLR(ADD) after 2 y & Selective muscle transfer \\
\hline Impaired pLR(ADD) over 4 y & Derotational humeral osteotomy \\
\hline
\end{tabular}

Abbreviations: a, active; ABD, arm abduction; ADD, arm adduction; LR, lateral (external) rotation; MRC, medial rotation contracture; p, passive; SSC, suprascapular. 
Table 2 Terminology for movements in lateral or medial rotation of the arm

\begin{tabular}{|l|l|}
\hline$p L R(A D D), a L R(A D D)$ & $\begin{array}{l}\text { Range of lateral rotation with } \\
\text { an adducted arm }\end{array}$ \\
\hline$p L R(A B D), a L R(A B D)$ & $\begin{array}{l}\text { Range of lateral rotation with a } \\
\text { 90-degree abducted arm }\end{array}$ \\
\hline$p M R(A D D), a M R(A D D):$ & $\begin{array}{l}\text { Range of medial rotation with } \\
\text { an adducted arm }\end{array}$ \\
\hline$p M R(A B D), a M R(A B D)$ & $\begin{array}{l}\text { Range of medial rotation with a } \\
\text { 90-degree abducted arm }\end{array}$ \\
\hline
\end{tabular}

Abbreviations: a, active; LR, lateral (external) rotation; MR, medial (internal) rotation; $\mathrm{p}$, passive.

Note: The clinically relevant movements are in italic.

Most of the muscle transfers to improve LR keep the transferred muscles (latissimus dorsi and teres major muscle) in a vertical line, ${ }^{16}$ so for evident biomechanic reasons they are aimed to improve aLR(ABD) and have less action on aLR (ADD). If one wants to improve aLR(ADD) specifically, the transferred muscle must have a horizontal pull line-this might be achieved in theory by rerouting the pectoralis major tendon, by a levator scapulae muscle transfer ( - Fig. 7), or by a 90-degree rotated, horizontalized teres major muscle body.

For all pre- and postoperative evaluations of aLR, a distinction between $\mathrm{LR}(\mathrm{ADD})$ and $\mathrm{LR}(\mathrm{ABD})$ is thus mandatory for both a proper surgical indication and any outcome analysis. Talking about active or passive lateral or medial rotation without specifying the arm position makes a great difference when analyzing the selective participation of one preexisting or transferred muscle in a specific movement in space. We therefore propose the following terminology for evaluation ( - Table 2 ) (only the five terms in italic have clinical significance). The range of $\operatorname{LR}(A D D)$ is important to describe the capacity to rotate outward and thereby the ability to flex the elbow with the arm maintained adducted (avoiding the trumpet sign), but maintaining pLR(ADD) in a range between 20 and 50 degrees is also an indicator for a satisfactory $\mathrm{GHJ}$ congruence. Considering active function, $\mathrm{aLR}(\mathrm{ABD})$ is the most important measurement (although in the Mallet score ${ }^{26}$ only aLR[ADD] is

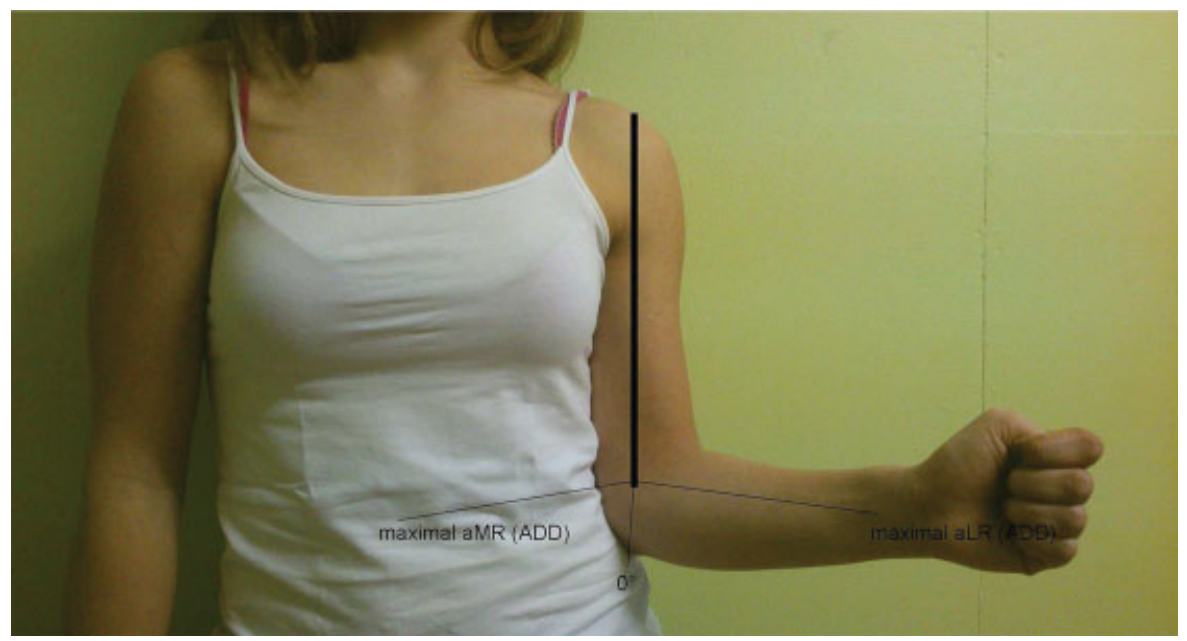

Fig. 3 LR in the horizontal plane with an adducted arm, that is, LR(ADD). The neutral position is at 0 degree, with the forearm put forward, the elbow flexed at 90 degrees. From this position, the range of MR and LR are shown.

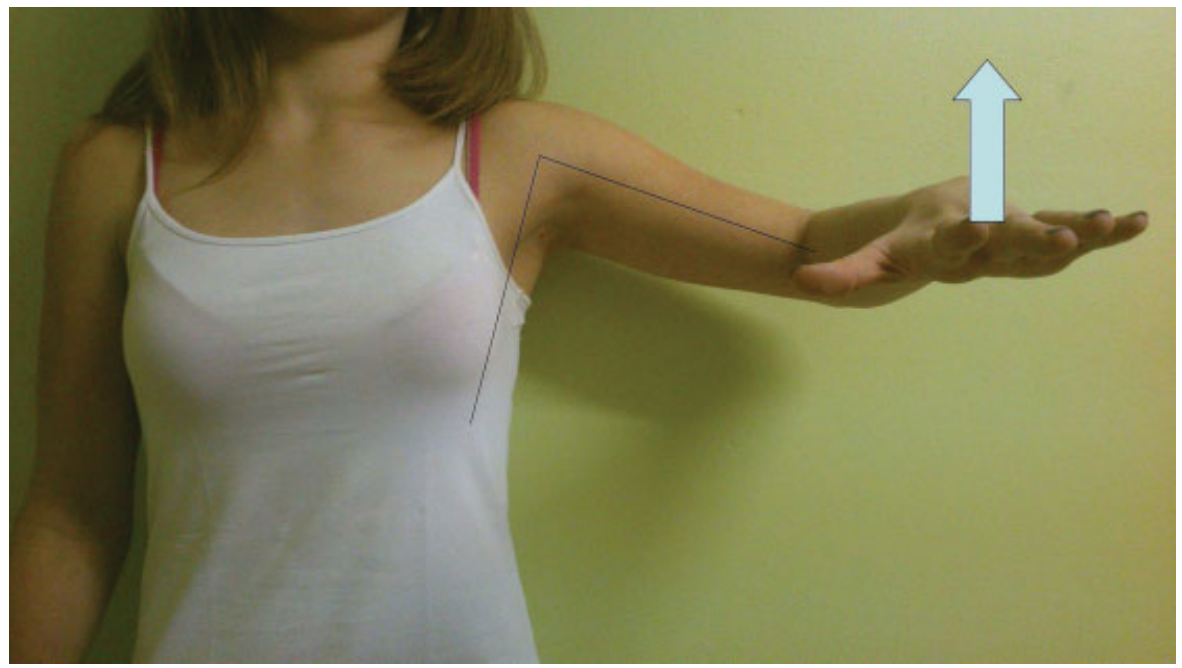

Fig. 4 LR in the sagittal plane with an abducted arm, that is, $L R(A B D)$. 


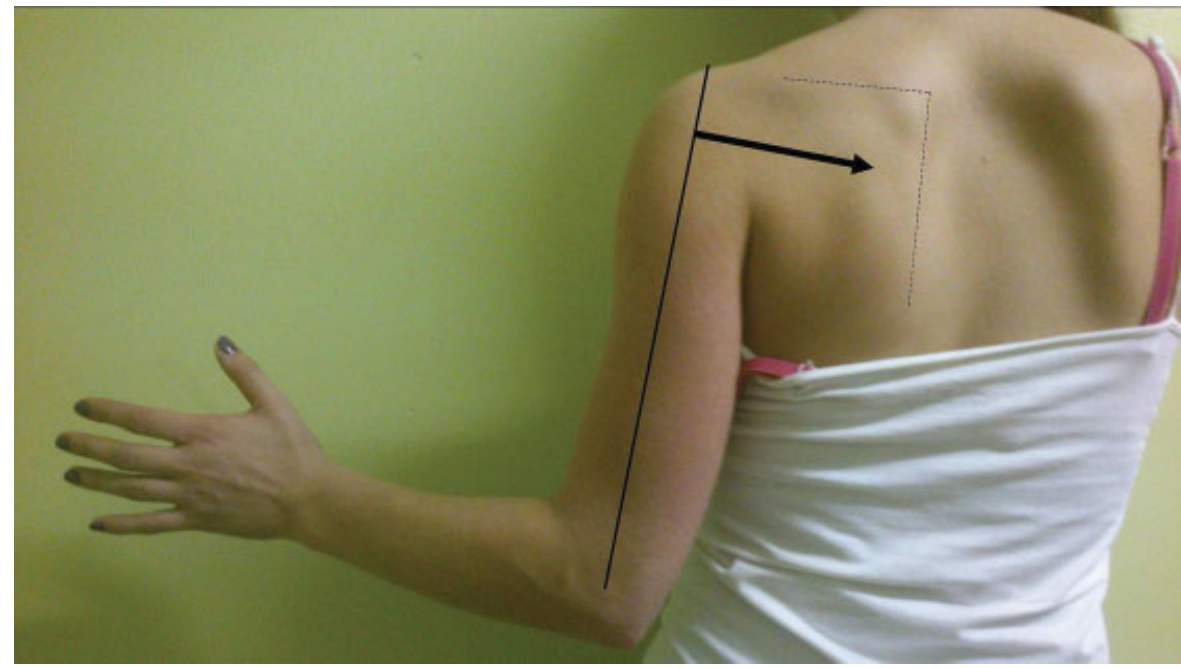

Fig. 5 aLR(ADD): the muscle contraction line is horizontal (arrow), perpendicular to the rotational axis (vertical line). The interrupted line shows the medial scapular contour.

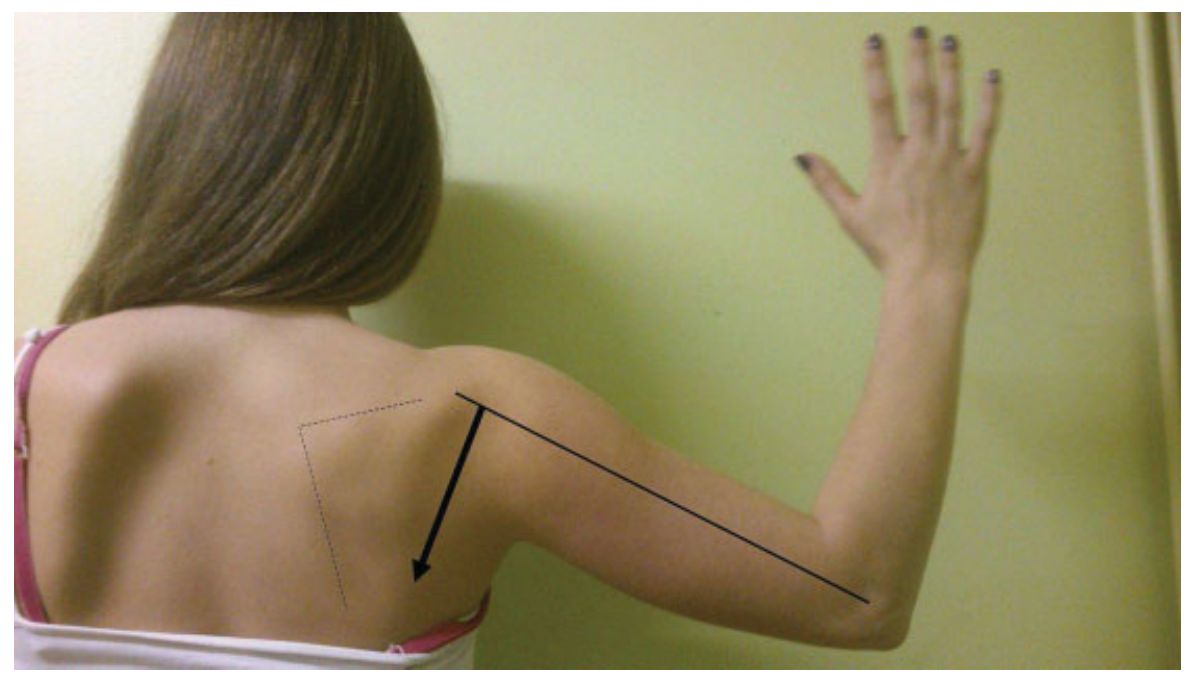

Fig. 6 aLR (ABD): the muscle contraction line is vertical (arrow), perpendicular to the rotational axis (horizontal line). The interrupted line shows the medial scapular contour.

represented in an own row), as this movement participates in overhead activities such as combing and dressing. MR(ADD) is an important measurement of medial rotation because it will objectivate drawbacks after an excessive surgical correction into LR (leading sometimes to external rotation contracture) and evaluates the function where the hand should reach the belly while dressing or cleaning the body.

\section{Discussion}

\section{Separation of Procedures Improving Passive and Active ROM}

Maintaining or restoring GHJ congruence to prevent GHD is the most important orthopedic rationale in MRC. In the first months of life, screening of posterior subdislocation of the humeral head by clinical means, conducted by pediatricians and/or specialized physiotherapists, with the same rationale of preventing hip dysplasia, and performing closed humeral head reposition under short general anesthesia are easy. Immobilization in LR(ADD) for
6 weeks does not interfere with the motor rehabilitation program. The follow-up after early reposition of the GHJ by closed or open reposition or subscapular muscle release using botulinum toxin shows up to $30 \%$ recurrence of subdislocation affecting 13 out of 37 cases in a recent study. ${ }^{27}$ The overall passive ROM of these joints with time seems to remain limited (own unpublished observations).

Any other surgical procedure aiming to improve motion in MRC should be composed of a minimum of defined steps with one target or clearly separated multiple targets, so that analysis of the pre- and postoperative function may be clearly related to the surgical step(s) performed. Also, surgery aimed to improve the passive ROM must precede one to increase active ROM, as muscle activity and strength may be hidden by restricted joint motion; and tendon transfers may not be evaluated postoperatively without considering the recovery of agonists. Regarding MRC in young children, a simple joint reposition or botulinum toxin application into the antagonistic subscapular muscle might reveal a recovering LR muscle 


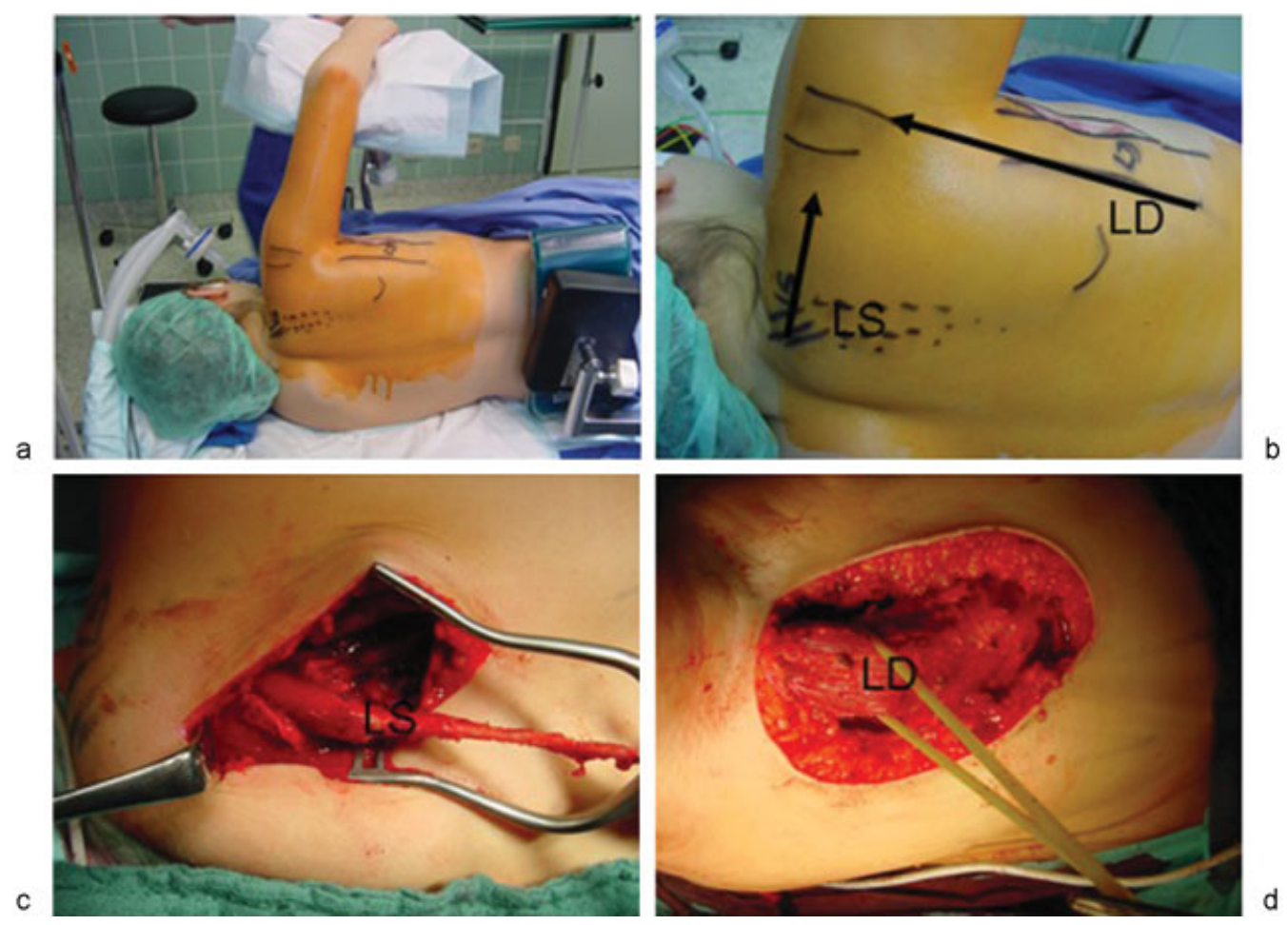

Fig. 7 Selective muscle transfers to improve LR. (a) A child in left lateral position, posterolateral approach onto the latissimus dorsi and levator scapulae muscles. (b) Closer view with pull lines for both muscles. (c) Harvest of levator scapulae muscle (LS) with a periosteal strip to lengthen the tendon, aimed to enhance aLR(ADD). This function may also be assumed by a teres muscle transfer with a horizontalized muscle body. (d) Harvest of latissimus dorsi muscle (LD) to augment aLR(ABD).

function, so far inhibited by the joint incongruence or soft tissue contracture. In a combined procedure (including the tendon transfer), the improvement of aLR thus cannot be attributed to the transferred muscle alone and the risk of surgical overindication is real, although the combined procedure of a joint release and tendon transfer may also result in a good functional improvement.

Moreover, while dealing with a rotational balance where two functional groups always interact, one may not focus only on improvement on one side (LR), while risking interference or decreased function on the other side (MR)-an aspect we will develop in the discussion on subscapular muscle weakening.

\section{The Role of Suprascapular Nerve Neurotization}

Any refinement in MRC treatment strategy must address the whole neuro-orthopedic sequence. Joint incongruence nowadays may easily be examined using ultrasound, if a skilled examiner is available for routine check-ups²; MRI may be added in older children with increased compliance. ${ }^{1}$ Concerning the rotational movement, the clinical importance of the recovery of the suprascapular nerve must be emphasized. There is no agreement about the timing when aLR should recover in a baby after BPBI or how late a suprascapular nerve and its muscles could be successfully reanimated by a nerve graft or transfer (van Ouwerkerk WJ, personal communication, 2012). ${ }^{23}$ Our policy to reanimate the suprascapular nerve as an isolated operation through a dorsal approach, in a child whose other key movements all recover, between 6 and 18 months, has been validated by our retrospective study. ${ }^{23}$ The clinical results in aLR(ABD) were much better than in aLR(ADD), but the latter still reached a mean of 29degree aLR(ADD) more than 3 years after surgery with the absence of significant GHD in this patient group, hypothesizing a protective effect on the $\mathrm{GHJ}$ congruence. Other studies showed that this nerve transfer is efficient. ${ }^{28,29}$ Unfortunately, this nerve transfer is still not included in recently published MRC management strategies. ${ }^{3,7}$ No publication states that significant dysplasia appeared in children with balanced rotation, either after spontaneous recovery or after suprascapular nerve reconstruction, unless we consider the rare cases of congenital humeral head (sub)dislocation. Early restoration of the rotational balance not only increases the functional score and the more "natural" shape of movements (elbow flexion without a trumpet sign), but also should allow a more congruent growth of the GHJ. Hence, one would expect less contracture around this important joint in the further growing child.

\section{Weakening the Subscapular Muscle}

MRC relates to an insufficiently compensated MR activity of the subscapular muscle. Its weakening might help to improve the rotational balance and also contribute to increase pLR (ADD), allowing better joint congruence. Early physiotherapy with stretching exercises of the subscapular muscle with the arm adducted is an important part of neurorehabilitation within the first 2 years. Botulinum toxin applied into this muscle is a regularly used adjunct ${ }^{27}$ when the muscle seems too strong and pLR(ADD) does not improve. Release surgery 
for MRC, either an anterior release ${ }^{14}$ or subscapular slide, ${ }^{15}$ is indicated to increase pLR(ADD) above 20 degrees and to reposition by surgical means a posteriorly (sub)dislocated humeral head, bearing the risk of decreasing aMR(ADD) by the lengthening tenotomy or muscle weakness through the subscapular muscle body slide. In this regard, it remains debated if the lengthening of the subscapular tendon (and the immediately visible forward motion of the humeral head into the anterior glenoid) effectively corrects a real subscapular muscle shortening or only acts through the resection of the upper, most scarred part of the fixed subscapular tendon. $A$ reduced pLR(ADD) is never found when the arm is abducted, where the $\mathrm{pLR}(\mathrm{ABD}$ ) remains (sub)normal, perhaps because the tilted scapula in shoulder abduction reduces the tension on the subscapular muscle by decreasing the distance from its scapular insertion to the humeral head.

MRC usually presents with reduced pLR(ADD) only, and we always start with an "anterior" $\mathrm{GHJ}$ release. The pathophysiologic role of the glenoid version and the surgical correction of a severe glenoid retroversion by a wedge osteotomy at the scapular neck including insertion of a bone graft are still very controversial.

A muscle transfer is planned and performed only when the joint motion range has been optimized and residual muscle activity had the chance to recover and to be assessed. We therefore advocate a minimum of 1-year delay with regular physiotherapy between surgical release and further decision regarding a muscle transfer for MRC and aLR weakness.

Finally, humeral derotational osteotomy is an ultimate solution in children with MRC, presenting GHD without chance of remodeling, posterior humeral head subdislocation associated with a severe glenoid deformity without hope of restoring a functional congruence, and sufficient aLR and aMR to increase function by shifting the ROM arc into more LR (generally $\sim 40-60$ degrees) without compromising reasonable aMR(ADD), bringing the forearm in front of the belly. ${ }^{30}$ However, this technique also may be considered as an alternative treatment with rather predictable outcome in younger children, to correct the arm position in either LR or MR. Also an osteotomy of the humerus could be combined with a tendon transfer aimed at increasing aLR.

\section{Functional Evaluation of aLR and Related Tendon Transfers}

For functional evaluation of the rotational balance at the shoulder level, the Mallet score ${ }^{26}$ remains the most widespread evaluation tool for functional shoulder movements in children suffering from BPBI. The second row of movements clearly evaluates lateral (external) rotation with the arm adducted (LR[ADD]), while the next row (hand to the nape of the neck) shows movements the child could execute by a consecutive abduction and elbow flexion, or by a LR with the abducted arm (LR[ABD]).

Our proposal on separation between the motion planes is not mandatory when a global functional assessment is performed. However, when postoperative outcome measurements are presented, and biomechanical arguments are discussed, the distinction between LR(ADD) and LR(ABD) becomes mandatory.
The available retrospective studies on the improvement of LR after obstetrical brachial plexus palsy (OBPP) with or without nerve or muscle transfer surgery unfortunately often do not mention which LR improved, unless they clearly relate to the drawings within the Mallet scale., ${ }^{9,16,31}$ Others report only on $\operatorname{aLR}(\mathrm{ADD})^{7}$ or $\mathrm{aLR}(\mathrm{ABD}),{ }^{3}$ thus being incomplete.

\section{From Patchwork to an Eclectic Strategy}

MRC in OBPP children presents with different symptoms at different ages. Also, our surgical procedures are so individual: different steps within an anterior release (amount of coracoid resection, section of coracohumeral ligament, amount of lengthening of subscapular tendon, associated proximal humeral MR osteotomy) or a muscle transfer (isolated or combined transfer of various muscles including latissimus dorsi, teres major, levator scapulae or pectoralis major; different tendon insertion points), or combination of both, associated or not with a subscapular weakening.

Therefore, further study designs should identify clearly defined variables in limited number about the indication and content of a treatment or surgical procedure. Motion assessment in children is sometimes hazardous, not repeatable, or impossible when the child is not compliant. ${ }^{2,9}$ For obvious ethic reasons, comparison with nonoperated control groups is rarely feasible in such a complex and impairing condition such as BPBI.

Meanwhile and even without more extended studies, the condition must be continued to be treated. Early recognition of joint incongruence or irremediable nerve lesion(s) is mandatory. Still surgical procedures may be optimized, when assessing muscle tension for a transfer, deciding about the precise tendon insertion point (defining the lever arm), and realizing this tendon fixation (with bone anchors or a transcartilaginous suture).

The evidence of "patchwork" studies combined with regular surgical practice under continuous improvement, associated with permanent clinical and scientific curiosity, should remain the base to MRC treatment strategy. Any surgeon involved in BPBI and MRC treatment should be aware of the whole diagnostic and therapeutic spectrum of this complex neuro-orthopedic condition.

\section{Conflict of Interest}

The author has no conflict of interest to disclose.

Note

This research received no specific grant from any funding agency in the public, commercial, or not-for-profit sectors.

\section{References}

1 Bahm J, Wein B, Alhares G, Dogan C, Radermacher K, Schuind F. Assessment and treatment of glenohumeral joint deformities in children suffering from upper obstetric brachial plexus palsy. J Pediatr Orthop B 2007;16(4):243-251

2 Bahm J. Changes in Rotatory Movements of the Shoulder after Obstetric Brachial Plexus Lesion: Clinical Condition, Surgery, and 
Analysis of Objective Prognostic Factors [thesis]. Brussels: Thesis ULB University; 2011

3 Abdel-Ghani H, Hamdy KA, Basha N, Tarraf YN. Tendon transfer for treatment of internal rotation contracture of the shoulder in brachial plexus birth palsy. J Hand Surg Eur Vol 2012;37(8):781-786

4 Cohen G, Rampal V, Aubart-Cohen F, Seringe R, Wicart P. Brachial plexus birth palsy shoulder deformity treatment using subscapularis release combined to tendons transfer. Orthop Traumatol Surg Res 2010;96(4):334-339

5 Ozben H, Atalar AC, Bilsel K, Demirhan M. Transfer of latissmus dorsi and teres major tendons without subscapularis release for the treatment of obstetrical brachial plexus palsy sequela. J Shoulder Elbow Surg 2011;20(8):1265-1274

6 Oztürk K, Bülbül M, Demir BB, Büyükkurt CD, Ayanoğlu S, Esenyel CZ. Reconstruction of shoulder abduction and external rotation with latissimus dorsi and teres major transfer in obstetric brachial plexus palsy. Acta Orthop Traumatol Turc 2010;44(3):186-193

7 Sibinski M, Hems TEJ, Sherlock DA. Management strategies for shoulder reconstruction in obstetric brachial plexus injury with special reference to loss of internal rotation after surgery. J Hand Surg Eur Vol 2012;37(8):772-779

8 Terzis JK, Kostopoulos E. Our experience with secondary reconstruction of external rotation in obstetrical brachial plexus palsy. Plast Reconstr Surg 2010;126(3):951-963

9 Thatte MR, Agashe MV, Rao A, Rathod CM, Mehta R. Clinical outcome of shoulder muscle transfer for shoulder deformities in obstetric brachial plexus palsy: a study of 150 cases. Indian J Plast Surg 2011;44(1):21-28

10 Bahm J, Elkazzi W, Schuind F. Forearm problems in obstetric brachial plexus palsy. In: De Smet L, Schuind F, eds. Difficult Problems and Complications at the Forearm. FESSH 2012 Instructional Course Book. Montpellier: Sauramps Medical; 2012

11 Soldado F, Benito-Castillo D, Fontecha CG, et al. Muscular and glenohumeral changes in the shoulder after brachial plexus birth palsy: an MRI study in a rat model. J Brachial Plex Peripher Nerve Inj 2012;7(1):9

12 Hultgren T, Einarsson F, Runesson E, Hemlin C, Fridén J, Ljung BO. Structural characteristics of the subscapularis muscle in children with medial rotation contracture of the shoulder after obstetric brachial plexus injury. J Hand Surg Eur Vol 2010;35(1):23-28

13 van Gelein Vitringa VM, van Kooten EO, Jaspers RT, Mullender MG, van Doorn-Loogman MH, van der Sluijs JA. An MRI study on the relations between muscle atrophy, shoulder function and glenohumeral deformity in shoulders of children with obstetric brachial plexus injury. J Brachial Plex Peripher Nerve Inj 2009;4:9

14 Birch R. Medial rotation contracture and posterior dislocation of the shoulder. In: Gilbert A, ed. Brachial Plexus Injuries. London: Dunitz; 2001:249-264

15 Carlioz H, Brahimi L. Place of internal disinsertion of the subscapularis muscle in the treatment of obstetric paralysis of the upper limb in children [in French]. Ann Chir Infant 1971;12(2): 159-167
16 Hoffer MM, Wickenden R, Roper B. Brachial plexus birth palsies. Results of tendon transfers to the rotator cuff. J Bone Joint Surg Am 1978;60(5):691-695

17 Dahlin LB, Erichs K, Andersson C, et al. Incidence of early posterior shoulder dislocation in brachial plexus birth palsy. J Brachial Plex Peripher Nerve Inj 2007;2:24

18 van der Sluijs JA, van Ouwerkerk WJ, de Gast A, Wuisman PIJM, Nollet F, Manoliu RA. Deformities of the shoulder in infants younger than 12 months with an obstetric lesion of the brachial plexus. J Bone Joint Surg Br 2001;83(4):551-555

19 Schmelzer-Schmied N, Ochs BG, Carstens C. Die Schulterluxation beim Neugeborenen. Bericht über 12 Fälle und Literaturübersicht. Orthopade 2005;34(5):454-461

20 Pauwels F. Gesammelte Abhandlungen zur funktionellen Anatomie des Bewegungsapparates. Berlin: Springer; 1965

21 Pauwels F. Atlas zur Biomechanik der gesunden und kranken Hüfte. Berlin: Springer; 1973

22 Kummer B. Biomechanik - Form und Funktion des Bewegungsapparates. Köln: Deutscher Ärzteverlag; 2005

23 Schaakxs D, Bahm J, Sellhaus B, Weis J. Clinical and neuropathological study about the neurotization of the suprascapular nerve in obstetric brachial plexus lesions. J Brachial Plex Peripher Nerve Inj 2009;4:15

24 Bahm J, Noaman H, Becker M. The dorsal approach to the suprascapular nerve in neuromuscular reanimation for obstetric brachial plexus lesions. Plast Reconstr Surg 2005;115(1):240-244

25 Blaauw G, Muhlig RS. Measurement of external rotation of the shoulder in patients with obstetric brachial plexus palsy. J Brachial Plex Peripher Nerve Inj 2012;7(1):8

26 Mallet J. Paralysie obstétricale du plexus brachial. II. Traitement des séquelles. Méthodes d'expression des résultats [in French]. Rev Chir Orthop Reparatrice Appar Mot 1972;58(Suppl 1):166-168

27 Ezaki M, Malungpaishrope K, Harrison RJ, et al. Onabotulinum toxinA injection as an adjunct in the treatment of posterior shoulder subluxation in neonatal brachial plexus palsy. J Bone Joint Surg Am 2010;92(12):2171-2177

28 Pondaag W, de Boer R, van Wijlen-Hempel MS, Hofstede-Buitenhuis SM, Malessy MJ. External rotation as a result of suprascapular nerve neurotization in obstetric brachial plexus lesions. Neurosurgery 2005;57(3):530-537, discussion 530-537

29 Tse R, Marcus JR, Curtis CG, Dupuis A, Clarke HM. Suprascapular nerve reconstruction in obstetrical brachial plexus palsy: spinal accessory nerve transfer versus C5 root grafting. Plast Reconstr Surg 2011;127(6):2391-2396

30 Al-Qattan MM, Al-Husainan H, Al-Otaibi A, El-Sharkawy MS. Longterm results of low rotation humeral osteotomy in children with Erb's obstetric brachial plexus palsy. J Hand Surg Eur Vol 2009; 34(4):486-492

31 Pagnotta A, Haerle M, Gilbert A. Long-term results on abduction and external rotation of the shoulder after latissimus dorsi transfer for sequelae of obstetric palsy. Clin Orthop Relat Res 2004;(426): 199-205 